\title{
Le reconditionnement de la perception dans un processus audio-phonatoire corrigeant le segmental par le suprasegmental : cas de la correction du son [y] chez l'apprenant marocain du fle.
}

\author{
Siham Azzi ${ }^{1,1}$, Souad El Yazidi ${ }^{2}$ \\ ${ }^{1}$ FLSHM, Laboratoire de recherches doctorales « langage, littérature et art », Maroc \\ ${ }^{2}$ FLSHM, Laboratoire de recherches doctorales « langage, littérature et art », Maroc
}

\begin{abstract}
Résumé. Qualifiées «Cendrillon » (Pistorius \& Bettina, 2011, p. 6) et faisant figure de " parent pauvre » de l'enseignement et de l'apprentissage des langues étrangères, la phonétique et la phonologie sont, aujourd'hui, de plus en plus marginalisées aussi bien dans le champ didactique que dans les pratiques de classe. Il en est de même pour la correction phonétique. Amener un apprenant à la production et la réalisation d'un son donné de la langue étrangère n'est pas toujours aisé pour l'enseignant. Jusqu'il n'y a guère, la plupart des enseignants de langue étrangère/seconde fondaient leur enseignement sur le postulat selon lequel l'apprenant prononce mal les sons étrangers parce que son appareil phonateur n'est pas habitué à les produire dans sa langue maternelle. La correction de la prononciation dans l'apprentissage d'une langue étrangère fait désormais consensus : faudrait-il rééduquer les organes articulatoires ou reconditionner la perception de l'apprenant (Alazard, 2013) afin de corriger ses déficiences de prononciation ? Un dilemme de causalité des erreurs phonétiques qui rejoint celui de " l'œuf ou la poule »? La présente étude expose les résultats de deux expérimentations portant sur la perception et la production de la voyelle orale [y] du français par des apprenants-enfants marocains commençant à apprendre cette langue à l'âge de 7 à 8 ans dans une école publique. Les tests de diagnostic conçus montrent que la voyelle antérieure arrondie $[\mathrm{y}]$, n'étant pas attestée ni en arabe marocain ni en arabe classique, pose majoritairement des problèmes de production aux apprenants marocains. Les résultats mettent en valeur l'efficience du reconditionnement perceptif sur les performances perceptive et productive des apprenants. Ils confirment que, généralement, un bon reconditionnement de la perception des sons étrangers est une nécessité préalable à une production optimale. Ces données obtenues suggèrent qu'un entraînement basé sur la correction perceptive par la prosodie sensibilise les apprenants aux liens existant entre le respect de la structure prosodique et la bonne perception et production des sons. Cela mène à conclure qu'une surveillance de la bonne maîtrise de la structure suprasegmentale dans l'expression orale, dès le début de l'apprentissage $\mathrm{du}$ français langue étrangère/seconde, pourrait garantir une meilleure performance chez l'apprenant.
\end{abstract}

\footnotetext{
${ }^{1}$ Azzisiham1@gmail.com
} 
Abstract. The reconditioning of perception in an audio-phonatory process correcting the segmental by the suprasegmental: case of the correction of sound $[y]$ in the Moroccan learner of the fle. Qualified as "Cinderella" (Pistorius \& Bettina, 2011, p. 6) and appearing as a "poor relation" in the teaching and learning of foreign languages, phonetics and phonology are, today, more and more marginalized both in the didactic field and in class practices. It is the same for phonetic correction. Bringing a learner to the production and realization of a given son of the foreign language is not always easy for the teacher. Until then, most teachers of foreign / second languages based their teaching on the assumption that the learner pronounces foreign sons badly because his phonetic device is not used to products in his language kindergarten.

Correcting pronunciation in learning a foreign language is now a consensus: does it require re-educating the articulation organs or repackaging the learner's perception (Alazard, 2013) in order to correct his pronunciation deficiencies? A dilemma of causality of phonetic errors that has joined that of "the chicken or the egg"?

This study presents the results of two important experiments on the perception and production of French oral vowel [y] by Moroccan child learners who learn this language at the age of 7 to 8 years in a public school. Diagnostic tests have revealed that the rounded anterior vowel [y], which does not exist in either Moroccan Arabic or Classical Arabic, poses mainly production problems for Moroccan learners. The results are highlighted the effectiveness of the perceptual reconditioning on the perceptual and productive performances of the learners. They confirm that, generally, a good repackaging of the perception of foreign sons is a prerequisite for optimal production.

This data provided suggest that no training based on perceptual correction by prosody makes learners aware of the links between respect for the prosodic structure and good perception and production of sounds. This leads to the conclusion that monitoring the good mastery of the suprasegmental structure in oral expression, from the start of learning French as a foreign language / second, could guarantee better performance for the learner. 


\section{Introduction}

La phonétique corrective est restée, depuis longtemps, un savoir qui n'a pas pu être transmis de façon adéquate "Le critère de la performance optimale (identité avec le système-cible) a été remplacé par le critère d'acceptabilité maximale des performances pourvu qu'elles n'entravent pas la communication »(Galazzi-Matasci \& Pedoya, 1983). Ignorer une erreur de prononciation dès le début de l'apprentissage ne contribue d'ailleurs qu'à augmenter son degré d'enracinement au fil du temps. Dans cet article, nous souhaitons nous consacrer à la question de la faisabilité de la transposition d'une correction phonétique en classe de langue seconde/étrangère. Nous proposons une étude dont le but est de montrer l'efficience de cette pratique sur l'amélioration des performances de production des apprenants. Cette étude, de telle sorte que d'autres très récentes (Sauvage, 2019), se veut un consensus cherchant à concilier l'approche communicative et la correction phonétique en classe de langue (Callamand, 1981) dans un contexte marocain. Il s'agira alors de se recentrer autour de questions neuroscientifiques puis psycholinguistiques, car il serait inconcevable de ne pas connaître le fonctionnement du mécanisme audiophonatoire avant de penser à instaurer une démarche psycholinguistique reposant sur le reconditionnement de la perception de l'apprenant afin de l'aider à percevoir et produire un son de la langue étrangère.

Aborder la perception, voire la reconditionner, n'est pas toujours un thème obvie pour les didacticiens et les enseignants. Il faut considérer qu'avec cette recherche, nous ne visons pas l'abstraction, mais de vérifier le degré de transférabilité d'un conditionnement optimale en classe de langue étrangère. L'objectif de cet article est bien évidemment de renouer les liens entre remédiation phonétique et didactique au sein d'une approche communicative et de vérifier aussi si la correction par la prosodie est une condition suffisante pour parvenir à percevoir le son [y] chez l'apprenant marocain. Pour cela, nous avons mené deux études expérimentales.

La première consiste à soumettre des sujets en une seule séance à la répétition de certaines approximations successives s'appuyant sur l'optimalisation suprasegmentale pour leur corriger la prononciation du son $[\mathrm{y}]$. La deuxième expérimentation, conduite sur une période d'entraînement basé en premier lieu sur des activités familiarisant l'apprenant aux régularités prosodiques et en second lieu sur des remédiations par des procédés suprasegmentaux dans les corrections in situ en classe, a pour objectif de rechercher l'efficience du conditionnement auditif sur la production de la voyelle [y] à la fin du parcours. Nous présenterons, avant tout, les hypothèses relatives aux principes des confusions des catégories des sons et à la correction phonétique, puis nous traiterons de leur application dans la didactique de l'oral pour faire suite à une approche psycholinguistique. Nous comparons a posteriori deux expérimentations dont l'objectif est de chercher l'apport du reconditionnement perceptif suprasegmental à l'habileté de prononciation du son [y]. 


\section{L'interférence langagière et le crible phonologique}

Les adeptes de l'analyse contrastive (Morlton, 1962; Lado, 1957; Ferguson, 1962-1966) et de l'hypothèse du filtre phonologique (Troubetzkoy, 1939) reconnaissent tous l'importance de l'origine linguistique de l'apprenant dans l'incarnation des habitudes articulatoires et prosodiques. D'un point de vue linguistique, Debyser (1970) considère l'interférence comme " un accident de bilinguisme» (Debyser, 1970, p. 34) résultant du contact entre les langues. Sur le plan psychologique, c'est «une contamination de comportements» (Debyser, 1970, p. 34). Ainsi, les assuétudes antérieures attribuent un effet négatif sur l'acquisition d'une nouvelle habitude. L'hypothèse de base est que l'interférence prédomine au début de l'apprentissage et disparaît progressivement en contribuant aux erreurs dues au développement. Le cas échéant, et si l'on se place dans une perspective pédagogique, la présence de ces interférences langagières nous mène à nous poser des questions sur les sources étiologiques de l'erreur de prononciation, sur quelles méthodes de correction phonétique nous devons nous baser pour porter aide à l'apprenant et sur le moment opportun pour apporter des remédiations. Quelles incidences donc de ces dernières sur l'acquisition des sons en langue étrangère (Alazard-Guiu, Santiago, \& Mariano, 2018) ?

Selon l'hypothèse de Troubetzkoy (1939), le système phonologique de la langue maternelle agit comme crible lors de l'apprentissage du système cible. L'apprenant est sourd à certaines sonorités, car son oreille n'est pas accoutumée à leur écoute : "les phonèmes de notre langue maternelle [...] se trouvent si étroitement liés avec notre activité perceptive que même en percevant des mots (ou phrases) d'une langue avec un système phonologique tout différent, nous sommes enclins à décomposer ces mots en des représentations phonologiques propres à notre langue maternelle » (Polivanov, 1931, p. 79). Bien que les deux systèmes phonologiques, de la langue maternelle et celui de la langue étrangère, soient définis et séparés, il naît de leur confrontation au début de l'apprentissage, un autre système : "le système de l'erreur » ou "le système de fautes" (Renard, 1979, p. 26). Le nouveau système de sonorité qui tend à changer un peu les habitudes audiophonatoires est en effet refusé à cause de ce crible phonologique. Étant donné que son oreille est conditionnée par son système maternel, l'apprenant perçoit donc les particularités des sons par rapport à ce conditionnement.

\section{La perception dans la correction phonétique}

La psycholinguistique et la psychologie cognitive ont démontré le rôle primordial des deux phénomènes d'audition et de phonation dans l'apprentissage d'une langue. S'intéressant aux systèmes mentaux mis en œuvre dans le traitement du langage, elles ont permis d'établir que la surdité phonologique est une conséquence de la perception catégorielle (Nguyen, 2005). Selon cette théorie, il est plus facile de percevoir les différences entre deux sons s'ils se rangent dans deux catégories phonétiques différentes plutôt que dans la même aire de catégorie. L'hypothèse de Kuhl (1991) nous explique aussi que l'espace perceptif se contracte autour d'un prototype fonctionnant comme un aimant qui attire vers 
lui les sons qui lui ressemblent sur le plan acoustique. Comme pour Troubetzkoy (1939), les modèles de Kuhl (1991), Flege (1995) et de Best (1994) confirment que les apprenants de L2 ne peuvent pas isoler les systèmes phonétiques de leur L1 et de leur L2. La proximité acoustique pour certains sons de L1 et de L2 rend l'assimilation plus difficile.

Le son de L2 est subséquemment mal prononcé, car les apprenants l'associent facilement à la même catégorie de L1 «Additionally, L2 sounds may be perceived as being new, similar, or identical to L1 sounds, and the degree of similarity or dissimilarity determines whether new L2 catgories can be establishd and/or whether equivalence classifications between the LE and L2 sounds may be made " (Zimpini \& Hansen Edwards, 2008 , p. 3). Selon une optique cognitive, Bagot aborde la perception comme désignant «l'ensemble des procédures qui nous permettent de prendre connaissance du monde environnant et de construire nos propres représentations mentales de ce monde. (...) implique de nombreux traitements au cours desquels l'individu interprète les informations issues de l'environnement et fonction de ses attentes et de ses motivations, compare à ses représentations une mémoire, prend des décisions, effectue des choix, se construit en somme une représentation du monde extérieur» (Bagot, 1999, p. 5). En résumé, la perception est une activité complexe et dynamique nécessitant l'intervention de nombreux mécanismes et processus. De là, il est de toute évidence qu'elle n'est pas limitée au sens de l'ouïe par la voie aérienne et il faudrait souligner davantage le caractère global de l'audition, la complexité du mécanisme au niveau du cerveau et l'aspect inconscient du processus perceptif.

\section{Le reconditionnement perceptif}

Dans l'apprentissage, le conditionnement est un processus qui allie le stimulus au comportement afin de façonner un nouveau comportement, réflexe dépendant des conditions du milieu entourant. Pavlov (1927) l'appelle le «réflexe conditionnel» ou la «réponse conditionnelle» et le considère comme étant primordial dans l'apprentissage chez l'être humain. Le processus de reconditionnement perceptif envisage de favoriser une catégorisation pertinente des nouvelles sonorités de la langue étrangère en préconisant des approximations successives qui permettent de se rapprocher graduellement de la prononciation visée.Le courant cognitiviste, basé sur le postulat selon lequel la correction phonétique repose principalement sur un travail de perception, considère que le processus audiophonatoire est un mécanisme non doué de contrôle conscient des organes articulatoires, mais d'un reconditionnement auditif perceptif du crible phonologique pouvant régénérer un réflexe articulatoire opportun (Boureux, 2017).

À l'opposé de ce courant, les méthodes articulatoires (désormais MA) sont fondées sur des coupes sagittales représentant la position du son-cible dans l'appareil phonateur et sur des descriptions des gestes articulatoires administrés au sujet ayant problème au niveau de ce son. Ces méthodes reposent essentiellement sur le postulat selon lequel «l'émission des sons implique une connaissance relativement poussée du fonctionnement de l'appareil phonatoire » (Guimbretière, 1994, p. 46). Dans cette perspective, l'enseignant doit avoir comme un savoir-faire la réalisation des différents phonèmes selon des représentations des mouvements articulatoires. Il doit utiliser des schémas afin de visualiser les diverses positions des organes du conduit vocal de l'appareil phonatoire. En revanche, Léon rappelle préalablement à ses descriptions articulatoires que «... le tort d'une description trop précise est surtout de laisser croire à l'élève que la position de la langue ou des lèvres se règle comme celle d'une commande mécanique et que le problème de la correction du son est alors résolu. Le processus articulatoire est plus complexe... » (1962, p. 20). Nous 
noterons donc que la MA néglige le facteur perceptif aussi bien que la dimension suprasegmentale tels que l'intonation et le rythme, facteurs primordiaux dans la production et la perception de la langue. Seuls les éléments segmentaux qui sont pris en considération rendant les perspectives de cette méthode plus ou moins cernées et réduites puisque les différents éléments de l'acte de communication : le geste et la prosodie ne sont pas tenus en compte.

Jones (2010) souligne également que «les plus subtils ajustements de la langue doivent se faire au moyen du contrôle sensoriel par l'oreille » et «le véritable contrôle est auditif» (Jones, 2010, p. 203). La langue étant considérée comme un comportement (Renard, 2010), nous pensons que toute intellectualisation et toute cérébralisation est à bannir lors des premiers stades de l'apprentissage. L'acte correctif, entendu dans un sens large comme l'ensemble de procédés déployés par l'enseignant, se doit donc de prendre en compte l'apport du reconditionnement de la perception à la performance de production des sons de la langue étrangère dans un long processus et avec un lent cheminement. Dans un sens plus restreint et s'inspirant de la méthode verbo-tonale, l'approche de remédiation in fine s'appuie sur l'optimalisation de la perception. Les principes de cette méthode soutiennent que la démarche instaure un processus de correction plus ou moins inconscient aidant l'apprenant à dépasser, faute de leur prononciation, le complexe psychique de blocage dans l'acte de communication.

La méthode verbo-tonale, fondée sur une stratégie interventionniste, plonge ses racines dans la psycholinguistique (Renard, 1979, p. 15) «La méthode V-T propose un ensemble structuré et systématique. Les procédés correctifs sont disposés de manière à reconditionner l'audition par une action portant non pas sur l'articulation du sujet, mais sur le modèle afin de procéder à une intégration inconsciente» (Guimbretière, 1994, p. 48) depuis (Landercy $\&$ Renard, 1977). Pour elle, reconditionner l'audiophonation c'est mener un acte correctif consistant à replacer l'élément fautif dans un environnement optimal, en recourant à la prosodie, à la phonétique combinatoire et/ou à la prononciation nuancée. Quant à la correction phonétique que nous proposons à notre public, elle se doit "une remédiation différée ou en temps réel » (Hamm \& Choi, 2018) par le truchement de diverses activités de répétitions, reposant sur le respect des régularités prosodiques, que les apprenants sont censés reproduire et restituer dans leurs activités de productions orales.

\section{La prosodie dans la correction phonétique}

Calbris et Montredon (1975), ces auteurs qui ont permis de rompre avec les méthodes correctives traditionnelles, ont ouvert une voie innovante et riche en didactique des langues. Ils ont montré l'importance de la prosodie dans la production orale : "La prosodie constitue aussi la trame phonétique d'une langue. Il y a solidarité entre les caractéristiques prosodiques et phonétiques. Par exemple, en français, l'accent de groupe détermine la régularité rythmique qui, par une égale répartition d'intensité et de durée, entraîne la netteté articulatoire de chaque phonème. Il est logique, dans ce cas, que l'acquisition des caractéristiques prosodiques d'une langue facilite celle des éléments phonétiques» (Calbris \& Montredon, 1975, p. 6). Pour eux, il n'est plus question de reléguer la phonétique à une simple activité de description articulatoire et à un exercice physique de l'appareil phonatoire, mais à des situations d'apprentissage qui, d'une part, soient en même temps des apprentissages encore plus complexes et qui, d'autre part, appartiennent au cadre du développement cognitif et comportemental de l'apprenant. Du point de vue de la 
méthode verbo-tonale l'idée défendue est que la correction par la prosodie positive l'attitude de l'apprenant face à la prononciation «il faut, dès le début de l'apprentissage, aborder le rythme, l'accent et l'intonation linguistique» (Lauret, 2007, p. 64).

Abry, D. \& Abry, J-V (2007) et Haidar \& Llorca (2016) confirment également que tout apprentissage d'une langue étrangère devrait accorder un intérêt à la prosodie dès le début. La question de l'importance de la mise en place d'une correction du segmental par le suprasegmental a été traité dans diverses études: (Di Cristo, 1999), (Fourgeron, 1998), (Vaissière, 1988), (Fernald, 1989), (Cortés, 2002) ont montré que la prosodie a un apport important à la prononciation des sons. D'autres chercheurs se sont intéressés à l'intégration des activités portant sur la reproduction fidèle de la structure prosodique des énoncés : Cornaz et al (2010) ont contribué à l'intégration de la voix chantée servant à la correction phonétique par la prosodie. Miras (2013) établit un consensus musique-parole et use des aspects prosodiques pour améliorer la performance phonétique de l'apprenant.

Le but de cette démarche corrective étant de permettre aux apprenants une concentration absolue sur la reproduction de la structure prosodique plutôt que de se focaliser sur un son. Nous avançons, en fait, que le problème de prononciation n'est guère exempt de difficultés articulatoires, mais plutôt d'ordre perceptif. La méthode de correction à adopter doit d'emblée insister sur l'importance de l'affectivité dans ce développement. Cette expérimentation vise, en somme, à mettre en place une intégration adéquate de la correction phonétique du segmental et du suprasegmental, dans les curricula de l'enseignement/apprentissage des langues étrangères, conséquemment par l'entremise des procédés suprasegmentaux. 


\section{Hypothèses}

C'est à partir des diverses réflexions citées auparavant que nous menons cette recherche. Cet article s'interroge tout d'abord sur les difficultés des marocains en termes de prononciation des sonorités du son [y] du français langue étrangère/seconde. Le protocole que nous adoptons porte sur deux expérimentations. D'une part, la première consiste à vérifier, l'efficience du reconditionnement de la perception sur la bonne production du son [y] chez les sujets-enfants commençant à apprendre la langue française. D'autre part, comme la méthode verbo-tonale est toujours assujettie à un travail lourd et ne donne pas de résultats rapides (Chabanal \& Mourier, 2019), nous trouvons qu'il est nécessaire de chercher l'apport de la correction par quelques approximations successives à l'habileté phonétique de l'apprenant. Pourrait-elle être optimale pour lui dans le hic et nunc quant à la discrimination du son [y] ? En s'appuyant sur une analyse contrastive, signalons d'emblée que la voyelle $[\mathrm{y}]$ du français n'est pas attestée en arabe.

Nous avançons donc que la plupart des sujets ne pourraient pas percevoir sa coloration facilement et par conséquent ils surestimeraient soit ses composantes sombres soit celles qui sont claires, et ils pourraient le confondre respectivement avec [u] ou avec [i]. La seconde expérimentation promeut une approche globale, intégrée, répondant aux objectifs assignés au préalable à l'enseignement du français langue étrangère dans une perspective multimodale imbriquant le versant perceptif et les autres dimensions de la pratique de l'oral. L'intégration d'une correction phonétique en classe de langue, suite à une démarche de lente maturité établie selon un processus implicite prenant en compte le respect des régularités prosodiques, permettrait- elle d'aboutir aux mêmes résultats que la première expérimentation ou instaurerait-elle davantage un développement de performances perceptives chez les apprenants?

\section{7. Étude empirique}

\subsection{Sujets}

Les sujets des deux expérimentations sont toujours les mêmes, des apprenants-enfants dont l'âge varie entre 6 et 8 ans commençant à apprendre le français langue seconde dans le curriculum scolaire institutionnel. Il n'existe pas de différence initiale entre eux au niveau de compétences langagières ou au niveau de situations sociolinguistiques. La classe se compose de 34 enfants-débutants des deux sexes.

\subsection{Expérimentation 1}

\subsubsection{Corpus et protocole de l'expérience}

Pour cette première expérimentation, nous avons conçu un corpus dont l'objectif est principalement de chercher les positions phonétiques où pourraient être générées les confusions phonétiques entre [y] et [i] et entre [y] et [u] chez l'enfant marocain commençant à apprendre le français langue étrangère, d'en expliciter les sources étiologiques puis de vérifier l'apport de la correction suprasegmentale à la correction du segmental. Nous avançons donc deux hypothèses. La première est que la confusion entre [y] avec [i] -ou-[u] dépendrait de sa position initiale, médiale ou finale de l'énoncé. La deuxième concerne la prosodie selon laquelle l'on pense que des approximations 
successives basées sur des optimalisations prosodiques favoriseront la correction implicite du son en question. Nous avons conçu et soumis six énoncés où nous avons pris le soin de mettre le son [y] en position initiale dans deux stimuli sous la forme d'une syllabe ouverte [v]. En position médiale et finale, existent deux énoncés pour chacune sous les deux formes syllabiques [cvc] et [cv]. Nous avons veillé à ce que tous les stimuli élaborés ne dépassent pas 3 à 4 syllabes pour répondre au principe de la mémoire de travail (Pisoni, 1975). Ainsi le corpus est présenté dans le tableau ci-dessous. Les énoncés constituent à la fois les stimuli du pré-test et le corpus de la procédure de correction.

Tableau. Stimuli du corpus.

\begin{tabular}{|l|l|l|}
\hline $\begin{array}{l}\text { [y] en position initiale } \\
\text { dans le stimulus }\end{array}$ & $\begin{array}{l}\text { [y] en position médiale dans le } \\
\text { stimulus }\end{array}$ & $\begin{array}{l}\text { [y] en position finale dans } \\
\text { le stimulus }\end{array}$ \\
\hline Unité trois (S3) & C'est une clé (S2) & C'est l'autobus (S1) \\
\hline Univers bleu (S4) & C'est du fromage (S5) & C'est la tortue (S6) \\
\hline
\end{tabular}

Le protocole de cette première expérimentation s'étend sur deux séances d'apprentissage filmées en deux jours dont le premier est consacré au test, déroulé en 15 minutes, sous forme de répétitions pour tous les 34 apprenants. Après avoir recensé les erreurs de prononciation, nous nous sommes préparées pour appliquer le lendemain du test une démarche de remédiation appropriée au type du diagnostic préalablement établi.

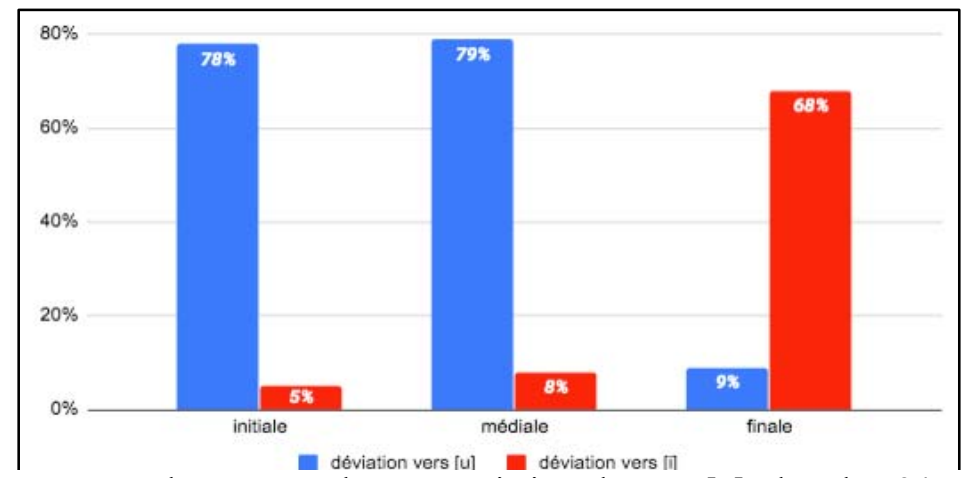

Fig. 1. Recensement des erreurs de prononciation du son [y] chez les 34 apprenants : Pourcentage des confusions de [y] avec [i] et [u]

\subsubsection{Protocole du test et résultats}

Le test consiste à inviter chaque apprenant à répéter l'énoncé que nous lui proposons. Tout d'abord, nous avons soumis aux apprenants les énoncés où le son [y] se trouve placé en position initiale. L'enseignant (toujours nous-mêmes) se place devant chaque apprenant, prononce l'énoncé et l'invite à le reproduire en désignant un geste lui cédant la parole. De ce fait, nous veillons à ce que la forme sonore de l'énoncé ne soit pas remplacée dans sa mémoire de travail par un autre mot de type 'vas-y' ou 'répète' à cause de l' « effet de récence » (Billières, 2002, p. 53). Une fois que le premier stimulus est répété par tous les apprenants d'une façon individuelle, nous recommençons la même opération avec le deuxième et ainsi de suite. Le corpus était testé en commençant par évaluer la 
prononciation de chaque stimulus de la position initiale, passant à ceux de la médiale et arrivant à la fin à ceux de la finale. Le graphique suivant représente les résultats obtenus du recensement des déviations trouvées dans la prononciation du son [y].

Les résultats révèlent que la voyelle [y] pose de majeures difficultés pour ces apprenants marocains. Sa déviance phonétique a un lien étroit avec sa position dans l'énoncé. L'observation des taux de déviations montre que la confusion entre [y] et [u] s'opère beaucoup plus en position médiale avec $79 \%$ et en initiale avec $78 \%$. L'apprenant, dans ce cas, surestime les traits sombres du son [y] et le confond avec [u] au détriment de sa coloration claire. En position finale, on note seulement $9 \%$ de cette confusion, mais une forte déviation vers [i] de $68 \%$ par effet, cette fois-ci, de surestimation de ses composantes claires. En position médiale $(8 \%)$ et en finale $(5 \%)$, [y] est moins confondu avec [i]. Nous remarquons que les résultats contredisent en quelque sorte nos prévisions. Suite au principe de l'optimalisation prosodique selon lequel sont présentés les stimuli, nous pensions qu'à la fin de la courbe descendante le son [y] en position finale serait perçu plus sombre et tendrait vers [u], mais la majorité l'a perçu [i] bien que cet aspect ne soit pas négligé pour autant.

Placé en début de la courbe mélodique, le son voit se valoriser son caractère clair et sera dévié par conséquent beaucoup plus vers le son [i], mais les résultats révèlent le cas contraire. En observant leurs prononciations de l'enveloppe sonore globale des stimuli, nous constatons généralement que l'accentuation ne s'établit pas au niveau de la dernière syllabe, mais à la pénultième, à l'antépénultième ou à l'attaque. La courbe intonative n'est plus respectée aussi. Faute de la scansion, la majorité déforme le rythme et réalise des durées syllabiques intempestives et produit un tempo saccadé. Il s'avère dès lors nécessaire de renforcer le travail sur le respect de la prosodie afin de pouvoir s'en servir dans l'assombrissement $\mathrm{du}$ son [y] en position finale et la mise en évidence de son éclaircissement en position initiale et médiale. La prise en compte de la remédiation optimale et idoine, avec un cumul de procédés prosodiques, se doit être dorénavant omniprésente en classe. 


\subsubsection{Protocole de la remédiation et résultats}

Pour remédier à ces erreurs de prononciation, il aura fallu que nous arrêtions une démarche fluide et beaucoup plus optimale par le biais de laquelle nous pourrions corriger la prononciation de $[y]$. De ce faisant, nous avons gardé le même corpus du test. Cela nous a permis de comparer les prononciations avant et après la remédiation comme présentées dans la figure 2. Étant donné que nos hypothèses sont fondées sur l'optimalisation des conditions de perception ou « la remédiation sur mesure » (Dańko \& Hamm, 2017), la démarche de la correction consiste, en premier lieu, à inviter chaque apprenant à répéter le stimulus S1(comme illustré dans le tableau ci-dessus). Puisque dans cette position le son [y] est confondu avec [i], l'apprenant apprécie donc son caractère clair et le met dans la catégorie de ce dernier. La correction consiste donc à assombrir sa prononciation pour permettre à l'apprenant de percevoir davantage le trait sombre de la voyelle. L'optimalisation de la perception se fait en prononçant par l'enseignant (nous-mêmes) ${ }^{\mathrm{i}}$ l'énoncé en intonation descendante en corrélation avec un geste de relâchement tout en accentuant davantage la dernière syllabe.

La macromotricité articulatoire occupe une place importante dans la correction (Billières, 2002). Dans le cas où l'apprenant trouve encore du mal à percevoir le son, nous compensons par d'autres répétitions optimalisées et par d'autres procédés accompagnateurs tels que la prononciation nuancée et les entourages facilitateurs, comme on peut recourir aussi au découpage progressif ou dégressif, etc., jusqu'à ce que l'objectif de la correction soit atteint. Une fois que les apprenants auront tous répété ce stimulus, nous passons aux répétitions du deuxième $\mathrm{S} 2$. Celui-ci est présenté en découpage progressif : il s'agit à présent d'inviter l'apprenant à répéter tout d'abord la séquence "c'est une" soumise aux mêmes conditions d'optimalisation que le précédent stimulus afin de pouvoir assombrir ou éclaircir la perception du son en fonction du sens de l'erreur. Ensuite nous lui demandons de répéter l'énoncé complet "c'est une clé". Pour le stimulus S3, nous proposons tout d'abord de reproduire "c'est une clé", déjà assimilé, en scansion syllabique pour avoir le stimulus « une clé » afin de mettre le son [y] au début de l'énoncé et le remplacer directement par le stimulus " unité trois".

Nous notons que pour cette première phase de correction, nous sommes allées dans un ordre dégressif, de la position finale à l'initiale. Comme la mémoire de travail est évanescente et l'information sonore ne demeure disponible que pour un très court terme, nous avons choisi d'enchaîner avec la dernière position optimalisée, c'est-à-dire S3, et de continuer la correction dans l'ordre suivant : répéter et corriger S4 pour tous les apprenants, de même pour S5 et enfin S6. Ainsi, nous constituons une sorte de boucle phonologique (Baddeley, 1986) au sein de laquelle se crée un magasin devenant réactif une fois un nouvel élément y arrive et y puise pour assurer la restitution. Nos procédés de correction sont fondés principalement sur l'optimalisation prosodique alliée au geste kinésique, mais dans le cas d'un crible phonologique réticent, nous accumulons avec la prononciation nuancée. Le décèlement des erreurs est illustré dans le graphique ci-dessous où nous présentons le nombre d'erreurs pour chaque stimulus, le nombre de corrections par la prosodie, les corrections optimales faisant appel aussi à la prononciation nuancée et le nombre de corrections non réussies nécessitant par la suite une prise en charge pédagogique adéquate à la nature des problèmes. 


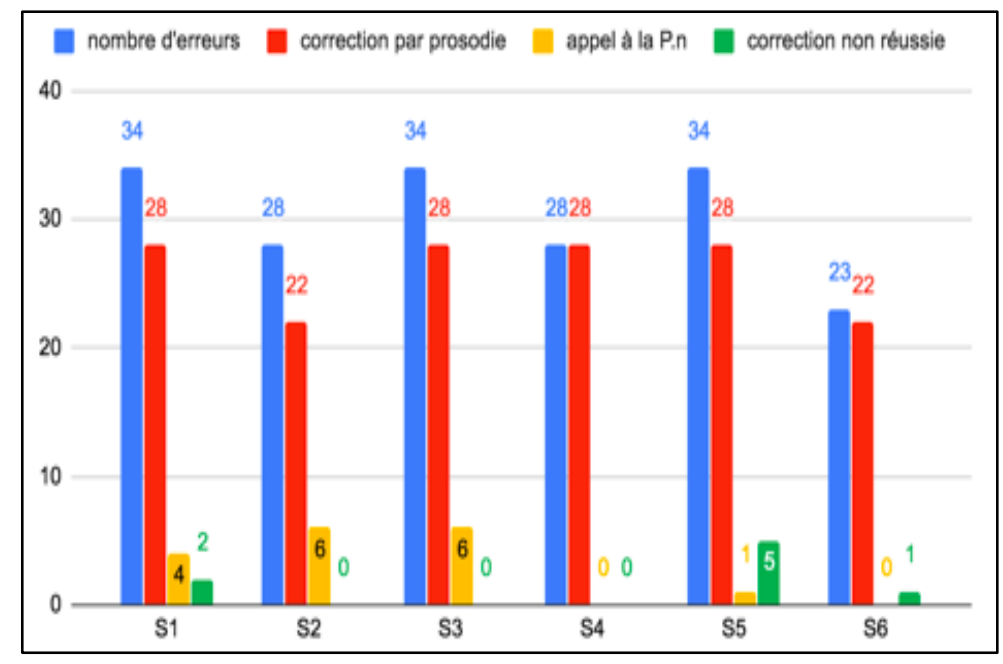

Fig. 2. Comparaison entre le nombre d'erreurs recensées, le nombre des corrections réussies uniquement par la prosodie et le nombre des cas interpellant le procédé de la prononciation nuancée (P.n)

Nous constatons que presque la majorité des erreurs sont corrigées sauf que certains apprenants avaient un crible phonologique tellement réticent qu'ils n'arrivent pas encore à assimiler le son dans $\mathrm{S} 1$, S5 et S6. D'après ces résultats, nous pourrions avancer que le procédé basé sur la régulation adéquate de la prosodie selon la nature de l'erreur était suffisant pour corriger la majorité des apprenants. Nous n'avons interpellé le procédé de la prononciation nuancée pour renforcer le timbre visé que dans 25 cas $(\mathrm{S} 1=6, \mathrm{~S} 2=6, \mathrm{~S} 3=6$, $S 5=6$ et $S 6=1$ ) sur 204 stimuli du corpus (6 énoncés $x 34$ apprenants) dont 17 réussis et 8 non réussis. 179 stimuli sont donc corrigés qu'avec le respect de la structure prosodique accompagnée d'un geste corporel. Nous observons d'ailleurs que le stimulus S4 est corrigé chez la totalité des apprenants uniquement avec une modulation intonative et kinésique. Les $\mathrm{S} 2$ et $\mathrm{S} 3$ sont bien assimilés par tout le monde. Les résultats révèlent, en fait, que la correction du segmental par le suprasegmental a répondu à l'objectif déjà assigné et a favorisé la perception du son [y].

Nous remarquons également que la perception des sujets est devenue plus affinée dans les stimuli S4, S5 et S6 corrigés en deuxième lieu après $\mathrm{S} 1, \mathrm{~S} 2$ et $\mathrm{S} 3$ qui ont nécessité plus de recours à la prononciation nuancée. Afin de vérifier l'efficience de l'optimalisation prosodique sur le débit d'appréhension de la bonne perception, nous voudrions relever les approximations successives ainsi que leurs cadences de recours dans l'affinement de la perception de $[y]$ dans chacun des stimuli. Rappelons qu'une approximation est une tentative de correction déployée grâce à un procédé optimal ou plus. Le graphique cidessous représente quatre modalités de remédiation en temps réel (Hamm \& Choi, 2018) en fonction de la capacité de réceptivité de chacun des apprenants. Pour certains cas, nous avons eu besoin d'une seule approximation ( 1 Appr). Dans le cas où si l'apprenant ne parvient pas à percevoir le son, nous lui ajoutons une deuxième approximation plus optimalisée ( 2 Appr) que la première afin d'accentuer davantage le timbre de la voyelle. Si pour les deux tentatives nous n'arrivons pas à mettre en relief la coloration du son et l'erreur persiste encore, nous ajoutons une troisième approximation plus adéquate $(>3$ Appr sans P.n). À ce stade, si nous avons tout essayé avec la prosodie, nous interpellons la prononciation nuancée ( $>3$ Appr avec P.n). Ainsi, nous obtenons les résultats suivants : 


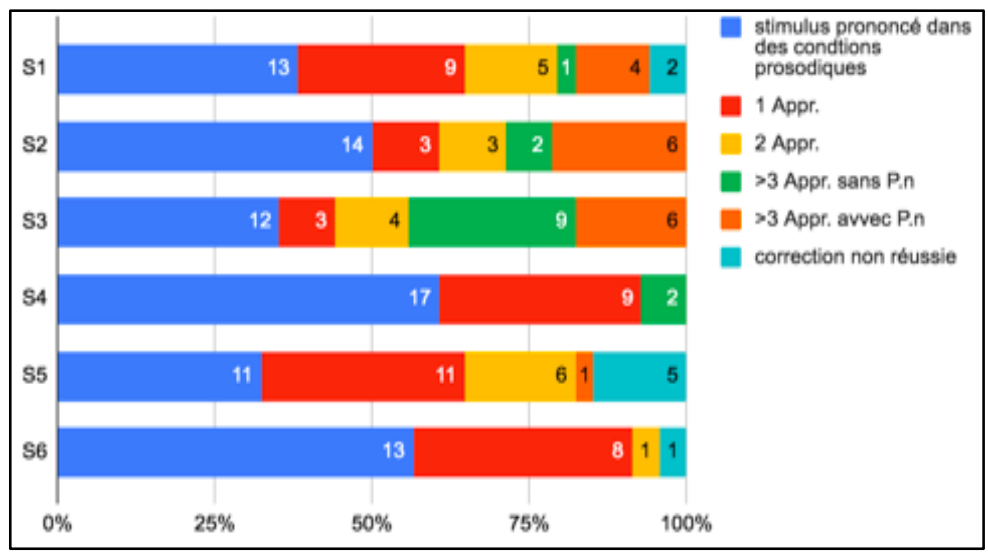

Fig. 3. Les approximations successives favorisant la perception du son [y] chez les apprenants.

Lors de la correction, la majorité des erreurs ont été corrigées uniquement par le recours à l'optimalisation suprasegmentale soit en proposant le stimulus dans la structure prosodique adéquate soit en ajoutant une approximation, deux approximations ou trois et plus. Pour certains cas, le crible phonologique est tellement réticent que nous avions fait appel au procédé de la prononciation nuancée pour mettre en relief davantage les traits voulus du son. Deux apprenants n'ont pas pu prononcer le son [y] dans l'énoncé S1 malgré le cumul de procédés exploités. De même pour 3 apprenants qui ont eu un vrai problème de perception du son dans le S5 et un seul ne pouvant pas prononcer correctement le son dans le S6.

Il faudrait noter que le recours à la prononciation nuancée (P.n) conjointe à plusieurs approximations était très important au début de la correction pour les trois premiers stimuli. La réduction du recours à plusieurs approximations dans les trois derniers stimuli révèle que l'assimilation du son commence à s'installer et le crible phonologique s'affine petit à petit chez les apprenants. Signalons aussi que le travail de correction par une seule optimalisation prosodique a réduit d'une façon satisfaisante la confusion du son $[\mathrm{y}]$ avec un autre, ce qui confirme le rôle important de la prosodie dans la correction phonétique.

Le fruit de cette étude est remarquable. La correction par la prosodie a donné de bons résultats sur le reconditionnement de la perception du son [y]. Même avec un tel travail réussi, les corrections ne sont pas prises comme une fin en soi, mais comme une phase préliminaire qui nous dévoile le rôle de la prosodie dans la correction et permet de se lancer dans une intégration d'un processus phonético-communicatif en classe de langue (Callamand (1981), Callamand \& Pedoya (1984), Guimbretière (1994), Billières (1995, 1996)). Comment deviendrait-elle donc la performance phonétique chez ces apprenants après une période d'intégration du travail phonétique correctif que par la prosodie? Tel est l'objectif de la seconde expérimentation.

\subsection{Expérimentation 2}

\subsubsection{Protocole du test}

Dans la perspective d'acquisition globale de la langue, il est impératif de s'appuyer sur une stratégie holistique. Il nous appartient en tant qu'enseignant, surtout au début de l'apprentissage, de prendre en considération le handicap que peut créer la surdité née du crible phonologique, qui contribue incontestablement à l'enracinement des autres cribles 
(culturel, syntaxique, lexical...). Dans cet objectif et après les résultats de l'expérimentation précédente, nous avons mené un travail correctif intégré dans le processus scolaire de ces mêmes apprenants en leur classe de langue. Le but est de prendre le soin d'opérer une assimilation phonétique du son dans une démarche s'appuyant sur « une lente maturation au niveau de l'inconscient» (renard, 2010, p165). L'intégration consiste à intervenir, chaque fois qu'une erreur se manifeste, par une correction par le procédé de la prosodie accompagné d'un geste allusif au sens de l'intonation adoptée ou un autre pour réguler le rythme, etc.. Les activités proposées, dans le dispositif pédagogique conçu, se basent généralement sur un climat dynamique et bienveillant comportant des exercices proches de la pratique théâtrale. Nous proposons également des répétitions aux apprenants pour les accompagner, leur faciliter l'écoute et principalement pour établir le changement désiré dans les habitudes de la prononciation.

Après douze séances hebdomadaires d'environ une heure d'enseignement du français, nous avons testé ces apprenants en leur proposant une activité sous forme de jeu de cartes. Les cartes contiennent chacune un dessin d'un objet dont le nom comporte le son [y]. L'enseignant présente toutes les cartes aux apprenants en leur expliquant leurs significations. Il demande ensuite à chaque apprenant de jouer contre lui. Possédant toutes les cartes dont les contenus sont cachés, il invite l'apprenant à en retirer une. Celui-ci donne le nom de l'objet en disant "c'est (nom de l'objet)". En principe, s'il le prononce bien il gagne la carte, sinon il la perde. Quand l'apprenant oublie ou se trompe dans la prononciation, l'enseignant tolère l'aide de ses camarades de classe ; vu que son objectif étant de faire prononcer le mot par chacun de tous les apprenants. Il veille en effet à ce qu'ils gagnent tous le jeu. Même un son, s'il est corrigé et bien prononcé, vaut une carte gagnée. Ce qui signifie que tout le monde gagnera toutes les cartes.

Lors de ces corrections, nous avons eu recours à deux modalités. En premier lieu, dans le cas d'une erreur, nous corrigeons uniquement en l'invitant, sans que nous prononcions la phrase, à la reproduire en suivant en même temps et proportionnellement la courbe mélodique désignée par notre geste macro-moteur ou notre attitude corporelle les plus adéquats à la nature de la déviance commise. Les mouvements de notre main sont synchrones in situ avec le continuum de l'énoncé. N'étant pas en mesure de percevoir le son, nous prononçons cette fois-ci l'énoncé corrélé toujours au même geste.

Le corpus de cette étude se compose de 14 phrases contenant chacune un mot comportant le son [y] précédé d'une consonne. Selon la phonétique combinatoire, les entourages de la voyelle influencent sur la perception de ses traits phonologiques, nous allons en effet évaluer la prononciation du son [y], chez nos sujets, quand il est précédé des consonnes suivantes : [s, f, R, n, m, b, d, z, 3, p, k, f, t, c]. Nous obtenons donc 476 stimuli. 


\subsubsection{Résultats et discussions}

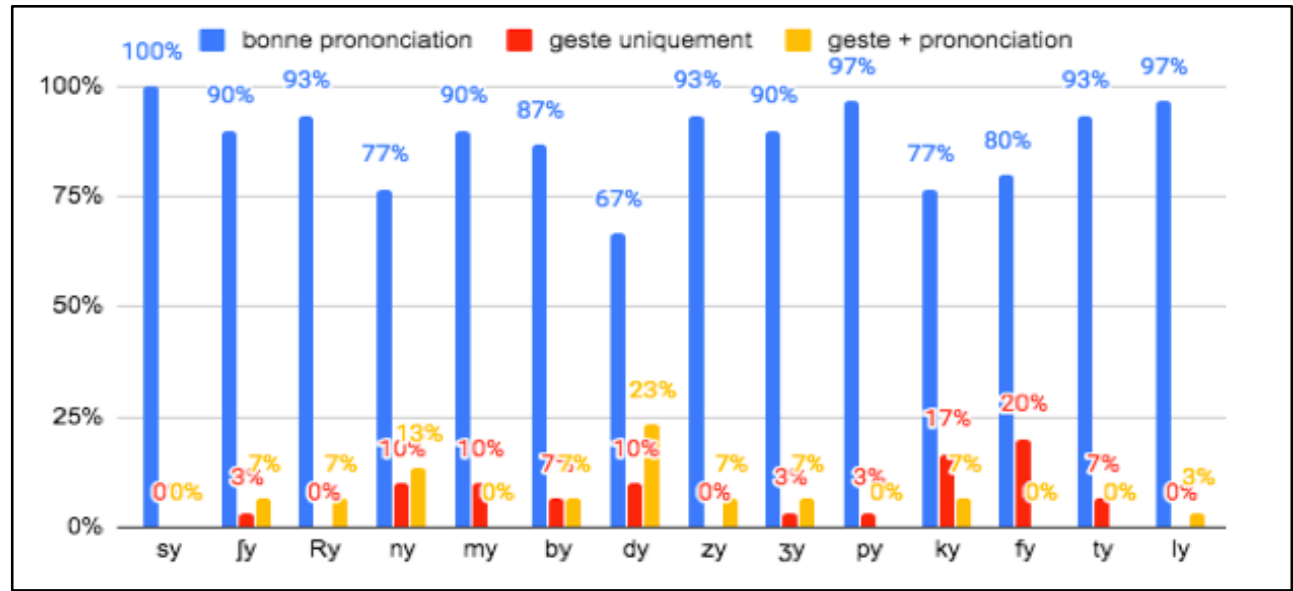

Fig. 4. Types d'interventions dans la correction à la fin du processus adopté : Résultats du post-test illustrant les deux aspects de la correction après l'intégration du travail correctif par la prosodie.

Le travail de correction intégré en classe semble être plus efficace sur la production des apprenants avec une baisse d'erreurs très remarquée comme illustrée dans le graphique cidessus. Les résultats montrent que l'enseignant n'a eu le besoin de recourir au geste (geste uniquement) que dans des cas très réduits ne dépassant pas $20 \%$ des apprenants surtout dans le stimulus contenant la syllabe [fy]. Quand l'apprenant n'arrive pas à corréler le geste avec la correction adéquate, l'enseignant l'aide avec une optimalisation verbale toute en prononçant le stimulus accompagné du geste approprié. Le taux d'intervention dans ces cas ne dépasse pas $23 \%$ dans le stimulus comportant la syllabe [dy]. Le taux de réussite de la remédiation varie entre $67 \%$ et $100 \%$ ne nécessitant absolument pas l'aide de l'enseignant. D'un taux d'erreur variant entre $77 \%$ à $87 \%$ au début de leur apprentissage (expérimentation 1) à un taux de bonne production avec ou sans aide de $100 \%$ et à un taux de quelques difficultés de perception entre $0 \%$ à $23 \%$ (expérimentation 2 , fin de l'apprentissage attribué) nécessitant légères interventions afin de le mener à s'autocorriger.

Selon notre seconde hypothèse, reconditionner la perception dans un processus intégré en classe améliore la production de [y] chez les apprenants. L'acte de solliciter un geste allusif référant à la structure suprasegmentale appropriée, au cours de la remédiation, est devenu un procédé suffisant dans l'autocorrection de l'apprenant. Le taux de recours à ce geste est très minime par rapport aux productions correctes des apprenants. Grâce à la correction par la prosodie, nous avons pu observer un progrès dans l'assimilation du son corrélé à un progrès sur l'autocorrection chez l'apprenant. Celui-ci, capable de se référer au geste et en effet à se corriger, est devenu très sensible au sens de la déviation erronée. Il a $\mathrm{pu}$ construire deux catégories différentes [y-i] et [y-u] là où il n'y en avait qu'une auparavant [i] ou $[\mathrm{u}]$. 


\section{Conclusion et perspectives}

S'approprier la langue comme une activité structurante, affective et inconsciente par hiérarchisation des approximations successives, toujours dans une globalité tout en instaurant des stratégies de conduites pédagogiques opératoires susceptibles de mettre en place des structurations dans un cours de langue (Renard, 2010, p. 290), favorise la sensibilité de l'apprenant à catégoriser les sons selon le système phonologique approprié.

Les premiers résultats de la première expérimentation augurent d'un certain affinement de la perception des apprenants et soutiennent l'apport du reconditionnement perceptif à l'habileté phonétique.

Il ressort de notre analyse que la variation de la confusion de [y] avec [i] ou [u] dépendait certes de la position phonétique, mais la complexité du phénomène de la perception ne nous permet pas de comprendre pourquoi dans la même position et dans le même stimulus certains sujets le perçoivent [i] et d'autres le confondent avec [u].

Même avec cette variation (Fourgeron, 1998; Colantoni, Steele, \& Escudero, 2008), que nous n'arrivons pas à expliquer empiriquement, les résultats ont montré qu'un reconditionnement adéquat à chaque type d'erreur peut la corriger quel qu'il soit son sens de déviation (plus sombre ou plus clair).

Cette démarche d'intégration de la correction phonétique en classe de langue a permis d'aboutir à certaines conclusions contribuant à l'amélioration de la didactique de l'oral :

-Reconditionner la perception est un protocole que l'enseignant pourrait intégrer en classe de langue.

-À l'opposé de la théorie articulatoire, reconditionner l'audition est un acte possible sans avoir point de peine de se préoccuper d'une analyse contrastive entre les systèmes phonologiques existant en confrontation chez l'apprenant et sans même chercher la source étiologique régénérant l'erreur de prononciation.

-Travailler sur la correction de la prononciation lentement et inconsciemment grâce à la prosodie débouche sur de bons résultats durables.

La posture corporelle était fortement omniprésente dans l'acte de correction et a contribué à l'instauration d'une attitude d'autocorrection chez les apprenants. Penser donc l'intégration de la posturomimogestuelle (Pavelin, 2002)et plonger l'acte de correction phonétique dans des situations pragmatiques et socioculturelles d'une interaction particulière rendraient sûrement la remédiation beaucoup plus fluide dans les échanges en face à face entre l'enseignant et les apprenants. 


\section{Bibliographie}

Abry, D., \& Abry, J.-V. (2007). La phonétique : audition, prononciation, correction. Paris: CLE International.

Alazard, C. (2013). Rôle de la prosodie dans la fluence en lecture oralisée chez des apprenants de Français Langue Etrangère. Toulouse: Thèse de doctorat, Université de Toulouse 2.

Alazard-Guiu, C., Santiago, F., \& Mariano, P. (2018). L'incidence de la correction phonétique sur l'acquisition des voyelles en langue étrangère : étude de cas d'anglophones apprenant le français. Aix-en-provence: Journéées d'Etudes sur la Parole, JEP 2018.

Baddeley, A. D. (1986). Working Memory. Oxford: Oxford University Press .

Bagot, J.-D. (1999). Information, sensation et perception. Paris: Armand colin, Coll. Cursus.

Best, C. (1994). Th Emergence of Native-Language Phonological Ifluences in Infants : A perceptual Assimmilation Model. Dans J. Goodman, \& H. Nusbaun, Th Development of Speech Perception : The Transition from Speech Sounds to Spoken Words (pp. 167-224). Cambridge: Mass MIT Press.

Billières, M. (2002). Le corps en phonétique corrective. In Renard, R. (dir.) Apprentissage d'une langue étrangère/seconde. Bruxelles : De Boeck Université (coll. Pédagogies en développement).

Boureux, M. (2017). Mieux percevoir pour mieux prononcer. Quelle phonétique corrective pour aider les apprenants italiens à bien parler français? dans le numéro spéciale Di Interculturel, rivista pubblicata dall'U,iversità del Slento ISBN 9788895343273 , 43-68.

Calbris, G., \& Montredon, J. (1975). Approche rythmique intonative et expressive du français langue étrangère. Paris: Cle International.

Callamand, M. (1981). Méthodologie de l'enseignement de la prononciation. Paris: Clé International.

Chabanal, D., \& Mourier, F. (2019). La question cognitive dans la phonétique corective : une approche exemplariste. Recherches en didactique des langues et des cultures, 16-1 .

Colantoni, L., Steele, J., \& Escudero, P. (2008). Second Language Speech. Theory and Practice. Cambridge University Press.

Cornaz, S. \&. (2010). L'apport d'exercices en voix chantée pour la correction phonétique en langue étrangère : le cas du français langue étrangère appliqué à des apprenants italiens d'âge adulte. Cahiers de l'APLIUT, 103-119.

Cornaz, S., Henrich, N., \& Vallée, N. (2010). L'apport d'exercices en voix chantée pour la correction phonétique en langue étrangère : le cas du français langue étrangère appliqué à des apprenants italiens d'âge adulte. Cahiers de l'APLIUT , 103-119.

Cortés, M. (2002). Didáctica de la prosodia del español : la acentuación y la entonación. Madrid: Edinumen.

Debyser, F. (1970). La linguistique contrastive et les interférences . Langue française, $n^{\circ} 8$, Apprentissage du français langue étrangère , 31-61.

Di Cristo, A. (1999). Le cadre accentuel du français contemporain : essai de modélisation. Langues, 2 (3) : 184-205 et Langues, 2 (4) , 258-267.

Ferguson, C. (1962-1966). Introduction générale à la série d'études contrastives . University of Chicago Press.

Fernald, A. (1989). Intonation and communicative intent in methers' speech tou infants : Is the melody the message? Child Development .

Flege, J. (1995). Second language speech learning: Theory, findings and problems. Dans W. Strange, Speech Perception and Linguistic Experience: Theoretical and Methodological Issues (pp. 233-277). Baltimore : York Press: Cross-Language Speech Research, York Press.

Fourgeron, C. (1998). Variations artiulatoires en début de constituants prosodiques de différents niveaux en français. Thèse de Doctorat de Phonétique, Paris: Université de Paris III - Sorbonne Nouvelle.

Galazzi-Matasci, E., \& Pedoya, E. (1983). Et la pédagogie de la prononciation? Le français dans le monde 180 .

Guimbretière, E. (1994). Phonétique et enseignement de l'oral. Paris: Didier.

Haidar, L., \& Llorca, R. (2016). L'oral par tous les sens. De la phonétique corrective à la didactique de l'oral. Recherches et applications-Le français dans le monde $n 60$.

Hamm, D., \& Choi, J. Y. (2018). Phonétique corrective en FLE : remédiation différée ou en temps réel, quelles priorités pour des apprenants hongrois et coréens? . Consécutivité et Simultanéité : en Linguistique, Langues et Parole-1. Phonétique, Phonologie, 89.

Jones, D. (2010). Dans R. Renard, Structuro-global et verbo-tonal variations 1962-2010 Essais de didactique des langues. Mons: CIPA. 
Kuhl, P. (1991). Human adults and human infants show a "perceptual magnet effect" for the prototypes of speech categories, mmonkeys do not. Perception ans Psychophysics, 50(2) , 93-107.

Lado, R. (1957). Linguistics accross Cultures. University of Michagan Press .

Landercy, A., \& Renard, R. (1977). éléments de phonétique. Paris-Mons: Didier-CIPA.

Lauret, B. (2007). Enseigner la prononciation du français: questions et outils. Paris: Hachette.

Léon, P. (1962). Laboratoire de langues et correction phonétique. Paris: Didier.

Miras, G. (2013). "Enseigner/apprendre" la prononciation autrement : une approche psychosociale musique-parole. Recherches en Didactique des Langues et des Cultures, Les cahiers de l'Acedle.

Morlton, W. G. (1962). The sounds of English and German. Contrastive Structure Series. University of Chicago Press .

Nguyen, N. \&. (2005). La perception de la parole. Phonologie et phonétique , 425-447.

Pavelin, B. (2002). Statut et rôle du mouvement dans la communication orale en face à face. Dans R. Renard, Apprentissage d'une langue étrangère/seconde 2. La phonétique verbo-tonale (pp. 71-87). Bruxelles: DeBoeck Université.

Pavlov, I. (1927). Conditioned reflexes. Oxford Univrsity Press. London .

Pisoni, D. (1975). Th role of auditory short-term memory in vowel perception. Memory \& Cognition, vol. $3,7-18$.

Pistorius, H., \& Bettina, W.-K. (2011). L'enseignement de la prononciation en langues étrangères. Babylonia 2, 4-89.

Polivanov, E. (1931). La perception des sons d'une langue étrangère. Travaux du Congrès de Prague. Den Haag 4.

Renard, R. (1979). Introduction à la méthode verbo-tonale de correction phonétique. Paris: Mons. Didier-Hatier. 3e édition.

Renard, R. (2010). Structuro-global et verbo-tonal variations 1962-2010 Essais de didactique des langues. Mons: CIPA.

Sauvage, J. (2019). Phonétique et didactique. Recherches en didactique des langues et des cultures, 16-1 .

Troubetzkoy, N. S. (1939). Principes de phonologies. Klincksieck.

Vaissière, J. (1988). prediction of velum movement from phonological specifications. Phonetica, vol. 45 , 122-139.

Zimpini, M.-L., \& Hansen Edwards, J.-G. (2008). Phonology and Second Language Acquisition. John Benjamins Publishing company.

${ }^{\mathrm{i}}$ Ayant des stages de formation en MVT 